\title{
Random Vibration Analysis for the Structure of the Airborne Evaporation Circulatory System
}

\author{
Yinsai Guo, Yi Zhang, Mengyi Zhang, Xiangfu Zeng \\ Department of graduate, Xijing University, Xi'an71012 , China, Email:guoysxj@163.com
}

Keywords: Airborne evaporation cycle system; Random vibration analysis; Modal analysis; PSD

\begin{abstract}
The structure of the airborne evaporation circulatory system is under the influence of the variety vibration caused by the helicopter during the long time running, which will cause the structure damage. The PSD analysis method is introduced into the random vibration analysis of the structure of the airborne evaporation circulatory system. A modal analysis of structure is carried out to obtain the modal frequency. When a acceleration PSD is loaded on the structure, stress and the dangerous position of the 1 sigma, sigma 2 and 3 sigma are obtained by adopted the software ANSYS Workbench. As the method is clear, simple calculation, it has a guiding significance for engineering application in design structure of airborne evaporation circulatory system.
\end{abstract}

\section{INTRODUCTION}

The structure of the airborne evaporation circulatory system is under the influence of the variety vibration caused by the helicopter during the long time running, which will cause the structure damage. The random vibration analysis mainly includes the time domain analysis method based on statistical counting and the frequency domain analysis method based on power spectral density[1-2]. Counting the number of cycles is the needed method and data processing is grate in the time domain analysis, while, it does not need to cycle count in the frequency domain analysis method. Based on the external turbulence of the system, the PSD of the stress response of the structure can obtained through dynamic simulation and finite element analysis. The structure dangerous point location can be got by using the PSD.

\section{BASIC THEORY OF POWER SPECTRAL DENSITY}

The power spectral density function is from the autocorrelation function by taking the method of Fourier Transformation in the stationary random process.

$S_{x}(\omega)=\int_{-\infty}^{\infty} R_{x}(\tau) e^{-j w \tau} d \tau$

The inverse transformation

$R_{x}(\tau)=\frac{1}{2 \pi} \int_{-\infty}^{\infty} S_{x}(\omega) e^{-j w \tau} d \omega$

The above two formulas make up the Fourier Transformation Spectromether, in which the autocorrelation function is:

$R_{x}(\tau)=E[x(t) x(t+\tau)]$

In practical engineering $f>0$, the unilateral power spectral density is defined:

$G_{x}(f)=\left\{\begin{array}{l}2 S_{x}(f) ; f \geq 0 \\ 0 ; f<0\end{array}\right.$

Spectral moments are introduced to describe the stochastic process digital characteristics of spectral density. The spectral moment of a smooth process $X(t)$ is defined by taking the method of unilateral spectral density $G$ (f):

$$
m_{n}=\int_{0}^{\infty} f^{n} G(f) d f
$$


The incidence of random signal with positive slope reaching the mean per unit time in the gaussian process:

$V_{a}^{+}=\sqrt{m_{2} / m_{0}}$

The incidence of random signal reaching the peak per unit time:

$$
V_{p}=\sqrt{m_{4} / m_{2}}
$$

\section{FINITE ELEMENT MODEL}

Structure material selection is Al7075; density is $2810 \mathrm{~kg} / \mathrm{m} 3$; the yield strength is $455 \mathrm{Mpa}$; safety factor is 1.5; allowable stress is $303 \mathrm{Mpa}$; the maximum deformation amount is not more than $1 \mathrm{~mm}$; elastic modulus is $71700 \mathrm{Mpa}$; poisson's ratio is 0.33 . According to the selected components, the weight of the condenser is $5.6 \mathrm{~kg}$; the weight of the compressor is $8 \mathrm{~kg}$; the weight of evaporator is $3.6 \mathrm{~kg}$. Bearing under distributed load of condenser supporting part is $2 \mathrm{x} 103 \mathrm{Mpa}$; bearing under distributed load of compressor supporting part is $1.4 \mathrm{x} 103 \mathrm{Mpa}$; bearing under distributed load of evaporator supporting part is $1.2 \mathrm{x} 103 \mathrm{Mpa}$; maximum load of air flow is 1.658 x103Mpa. Boundary condition is shown in figure 1.

Mesh is automatically divided which is shown in figure 2. Node number is 13790; element number is 3984.

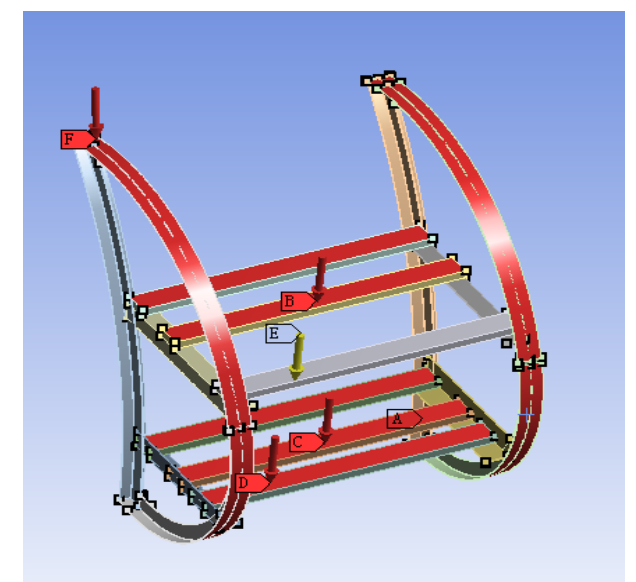

Fig.1 Boundary condition

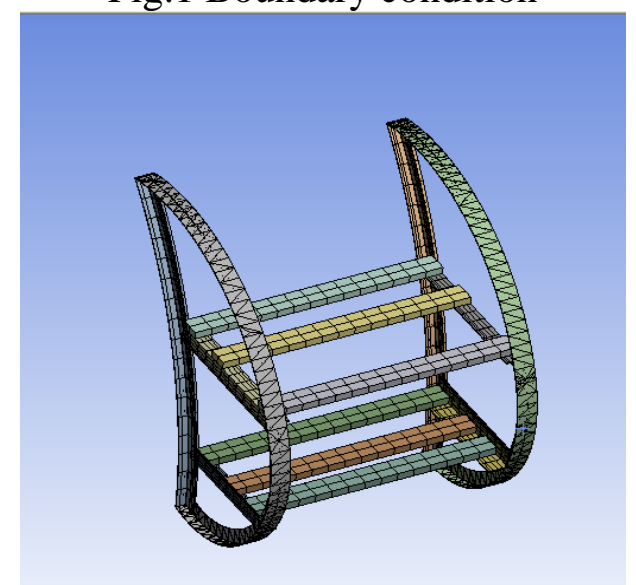

Fig.2 Mesh chart

\section{MODAL ANALYSIS}

Modal analysis is mainly to understand the modal frequency and vibration model of the structure[3-4]. Each order natural frequency covered in frequency band of the PSD load spectrum is got to prepare for the next step of the random vibration analysis. Calculate the first 20 frequencies shown in figure 4. 


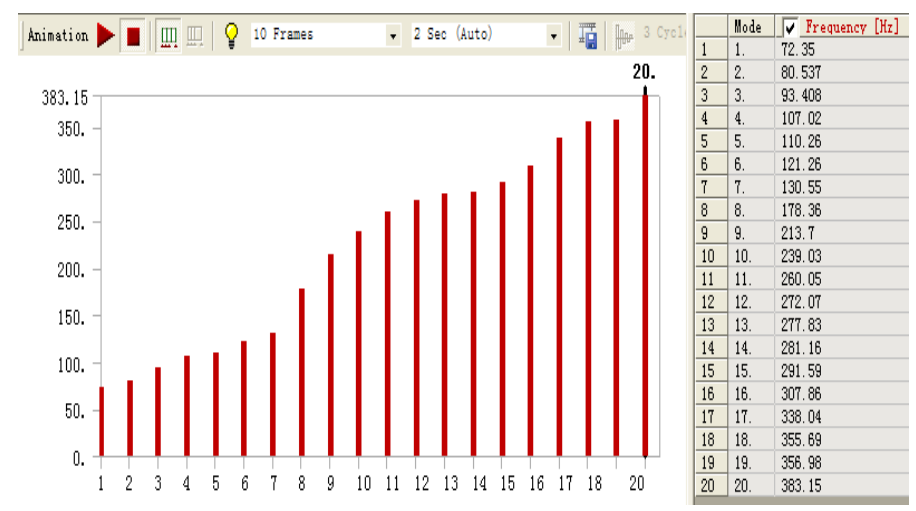

Fig.3 The acceleration power spectrum

\section{RANDOM VIBRATION ANALYSIS}

Taking the random vibration load spectrum of the helicopter-the PSD acceleration power spectrum as an incentive[5]. To ensure that all frequencies of the modal analysis can be used, the frequency ranges from $10 \mathrm{~Hz}$ to $2000 \mathrm{~Hz}$. The PSD power spectral density function is inputted in the ANSYS Workbench which is shown in figure 5.

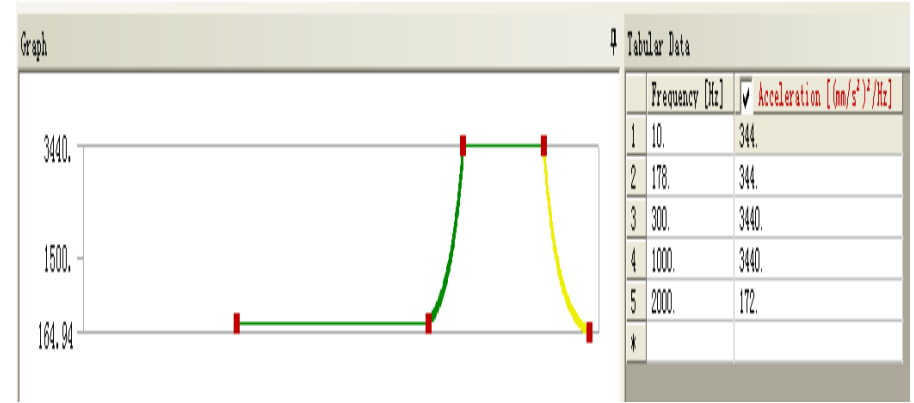

Fig.4 The acceleration power spectrum

The values of maximum stress and deformation and the position of the dangerous point can be attained at the level of 1 sigma, 2 sigma, and 3 sigma.

(1) The stress value is $0.719 \mathrm{MPa}$; the deformation value is $0.011 \mathrm{~mm}$; the dangerous point occurs on the support pieces of the condenser at the level of 1 sigma, which is shown in Figure.5.

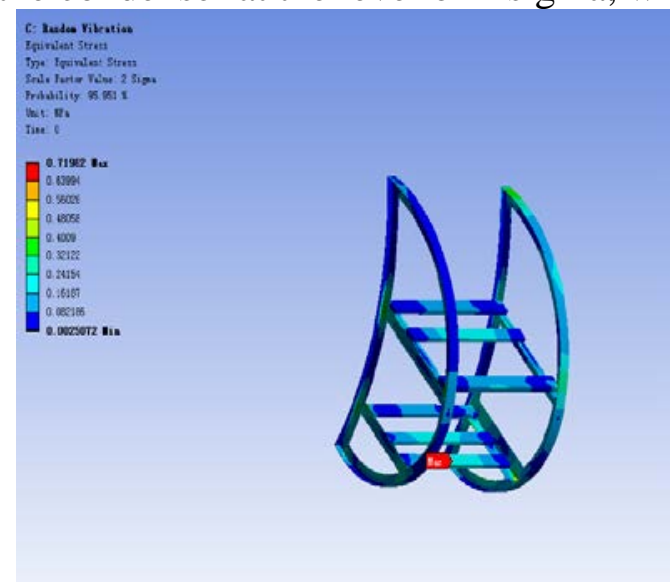

(a) Deformation 


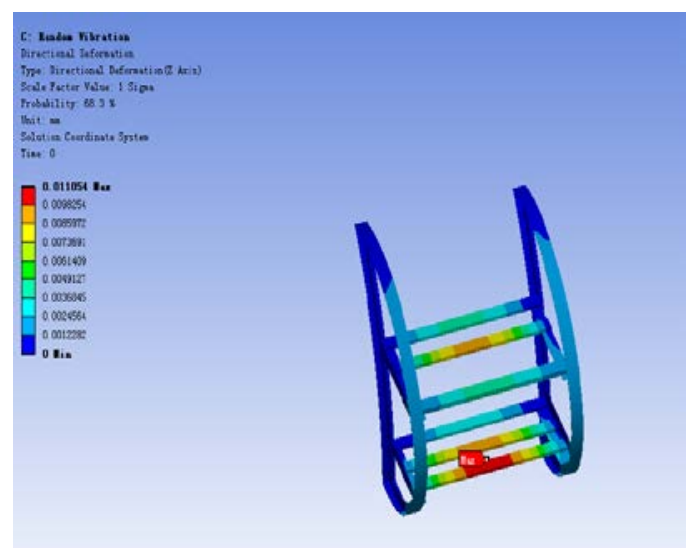

(b) Stress

Fig. 5 Stress and deformation results of 1sigma level

(2) The stress value is $0.719 \mathrm{MPa}$; the deformation value is $0.022 \mathrm{~mm}$; the dangerous point occurs on the support pieces of the condenser at the level of 1 sigma, which is shown in Figure.6.

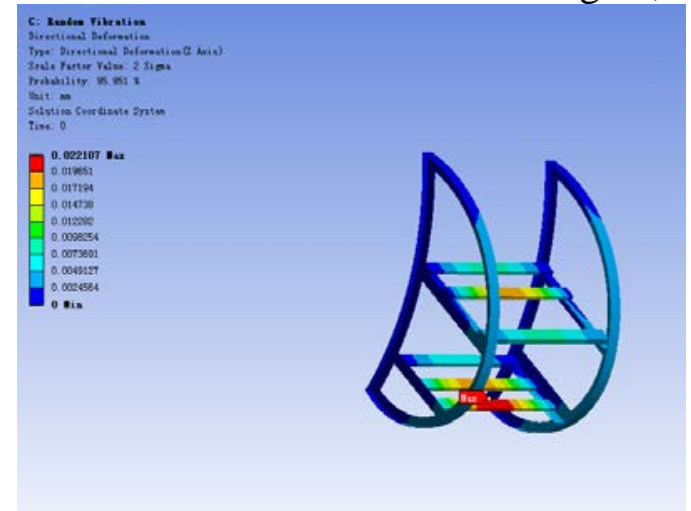

(a) Deformation

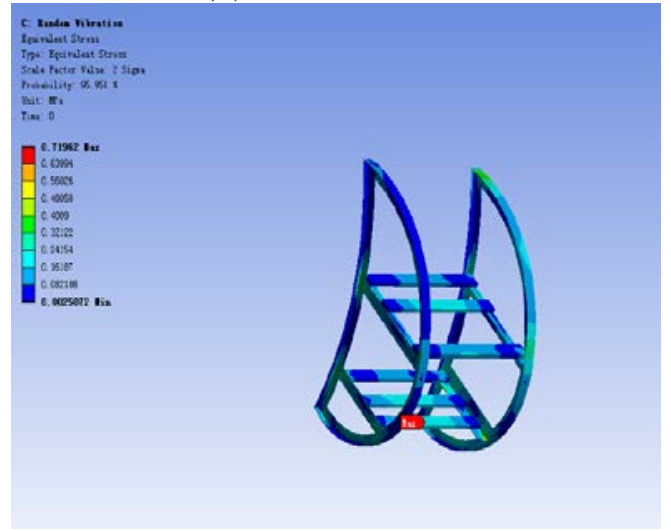

(b) Stress

Fig.6 Stress and deformation results of 2sigma level

(3) The stress value is $1.709 \mathrm{MPa}$; the deformation value is $0.033 \mathrm{~mm}$; the dangerous point occurs on the support pieces of the condenser at the level of 1 sigma, which is shown in Figure.7. 


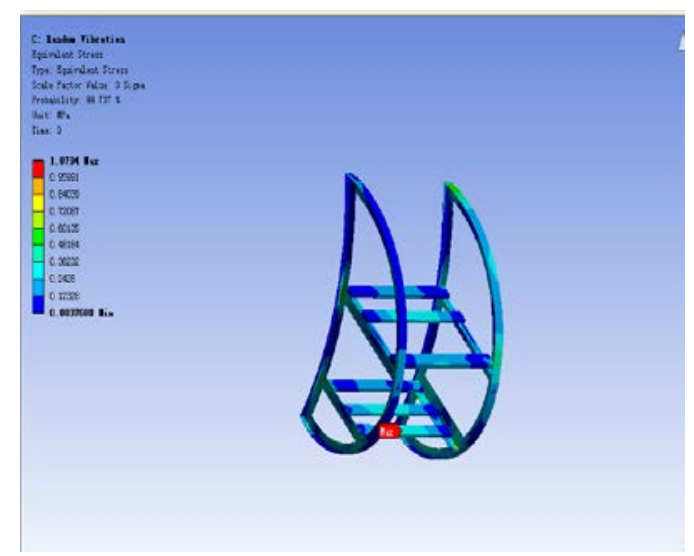

(a) Deformation

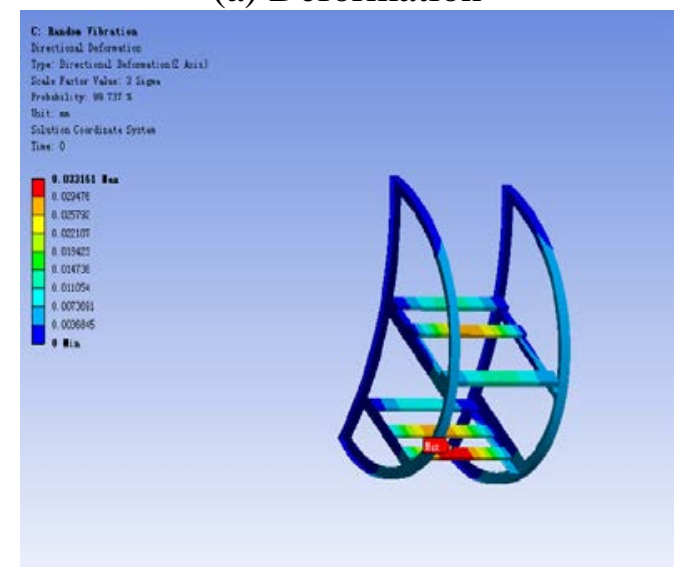

(b) Stress

Fig.7 Stress and deformation results of 3sigma level

As can be seen from the above, the dangerous point occurs on the support pieces of the condenser; the stress meets the requirements of the allowable stress and the deformation is less than $1 \mathrm{~mm}$. So the designed structure can meet the requirements.

\section{CONCLUSIONS}

The PSD analysis method is introduced into the random vibration analysis of the structure of the airborne evaporation circulatory system. The stress and deformation in different probability can meet the design requirements, and the dangerous position of the structure is determined. Greatly improve the efficiency of design and provide guiding significance for engineering applications of the airborne evaporation circulatory system structure.

\section{ACKNOWLEDGEMENTS}

This work was supported by the Natural Science Foundation of Shaanxi Province (Program No. 2013JM8040), the Scientific Research Program Funded by Shaanxi Provincial Education Department (Program No. 2013JK1204) and Graduate Student Innovation Fund of Xijing University.

\section{REFERENCES}

[1] Zhao-xu Li. The vibration design of electronic equipment[J]. Electro-Mechanical Engineering, 2002, 18(1): 51-55.

[2] Yu-jun Yang. ANSYS dynamics simulation technology in the application of the space structure design for computer cases[J]. Electro-Mechanical Engineering, 2003, 19(5): 42-46. "In Chinese"

[3] Jian-feng Ren, Chou Yuanying, Duan Baoyan. A new simulation method based on the acceleration impact[J]. Modern Machinery, 2006, (2): 20-26. "In Chinese"

[4] Xing-min Wang. Finite element analysis of structure of locomotive air-conditioner [D]. 
Shijiazhuang Tie dao University, 2013. "In Chinese"

[5] Yong-chao Fang. Stochastic fatigue analysis method of ANSYS [J], Information Technology, 2009,(21): 19-23. 